\title{
Streikrecht auch für Beamte?
}

\author{
Eine Auseinandersetzung mit dem Streikverbot für Beamte unter \\ Berücksichtigung der Rechtsprechung des EGMR
}

Das Beamtenstreikverbot ist ein Urgestein des deutschen Berufsbeamtentums. Die deutsche Rechtsordnung befindet sich infolge der Europäisierung in einem Wandel. Davon sind selbst die hergebrachten Grundsätze des Berufsbeamtentums nicht ausgenommen. Angesichts der aktuellen Entscheidung des Verwaltungsgerichts Osnabrück $^{1}$ und der widerstreitenden Entscheidung des Verwaltungsgerichts Düsseldorf im letzten $\mathrm{Jahr}^{2}$ soll in diesem Beitrag das Beamtenstreikverbot unter Einbeziehung des Streikrechts nach Art. 11 EMRK und der dazugehörigen Rechtsprechung des Europäischen Gerichtshof für Menschenrechte (EGMR) untersucht werden.

\section{Einführung}

Die Ausübung hoheitsrechtlicher Befugnisse als ständige Aufgabe ist laut Art. 35 Abs. 4 GG in der Regel Angehörigen des öffentlichen Dienstes zu übertragen, die in einem öffentlich-rechtlichen Dienst- und Treueverhältnis stehen. Das Recht des öffentlichen Dienstes ist gemäß Art. 33 Abs. 5 GG unter Berücksichtigung der hergebrachten Grundsätze des Berufsbeamtentums zu regeln und fortzuentwickeln. Diese Grundsätze beinhalten den Kernbestand von Strukturprinzipien, die während eines längeren, traditionsbildenden Zeitraums als verbindliche Grundsätze anerkannt worden sind. ${ }^{3}$ Nach der ständigen Rechtsprechung des Bundesverfassungsgerichtes (BVerfG) und Bundesverwaltungsgerichtes (BVerwG) gehört die Treuepflicht seit jeher zu den Kernpflichten des Beamten. Daraus wird das Streikverbot für die Beamten hergeleitet. ${ }^{4}$

Der Beamte ist dem Allgemeinwohl und damit zu uneigennütziger Amtsführung verpflichtet und hat bei der Erfüllung der ihm anvertrauten Aufgaben seine eigenen Interessen zurückzustellen. Der Einsatz wirtschaftlicher Kampf- und Druckmittel zur Durchsetzung eigener Interessen, wie das Streikrecht nach Art. 9 Abs. 3 GG, sind ihm verwehrt. $^{5}$

Ein Streik als eine Form von Arbeitskampf würde sich gegen den Gesetzgeber richten ${ }^{6}$ denn die Gehälter der Beamten werden nicht im Wege der Verhandlungen, sondern der Gesetzgebung festgelegt.

1 VG Osnabrück, Urt. v. 19.8.2011 - 9 A1/11 und 9 A 2/11.

2 VG Düsseldorf, Urt. v. 15.12.2010 - 31 K 3904/10.0.

3 Pieper, in: Schmidt/Bleibtreu/Hoffmann/Hopfauf, 12. Auflage (2011), Art. 33. Rdnr. 112.

4 Jarass, in Jarass/Pieroth, 11. Auflage (2010), Art. 9, Rdnr. 56.

5 BVerfGE 8, 1 (17) = NJW 1958, 1228; BVerwGE 73, 97 (102) NJW 1981, 1283; BVerwGE 69, $208(212)=$ NJW 1984, 2713.

6 Badura, in Maunz/Dürig, 62. Lieferung (2011), Art. 33 GG, Rdnr. 58. 
Während das Tatbestandsmerkmal „hoheitliche Befugnisse“ des Art. 33 Abs. 4 GG immer wieder kontrovers diskutiert wird - gemeinhin um eine weitere Ausnahme zugunsten der Privatisierung zuzulassen -, rückt die herrschende Meinung in Deutschland von dem absoluten Verbot des Streiks für Beamte nicht ab. Der Europäische Gerichtshof für Menschenrechte ist jedoch anderer Ansicht. Der EGMR hält ein generelles Verbot des Beamtenstreiks mit dem Art. 11 EMRK für nicht vereinbar. ${ }^{7}$ Damit besteht eine Divergenz zwischen der Rechtsprechung des EGMR und der des Bundesverfassungsgerichts. Was diese Divergenz zur Folge hat, bringen die antagonistischen Entscheidungen der Verwaltungsgerichte Düsseldorf und Osnabrück am besten ans Licht. Zur Aufdeckung der sich hieraus ergebenden Probleme wird zunächst das Streikrecht nach Art. 11 EMRK und die dazu gehörige Rechtsprechung des EGMR erörtert und deren Wirkung und Bedeutung für das nationale Recht herausgearbeitet. Schließlich werden die Entscheidungen der Verwaltungsgerichte Osnabrück und Düsseldorf in den Blick genommen.

\section{Das Streikrecht nach Art. 11 EMRK}

Art. 11 Abs. 1 EMRK schützt die Versammlungs- und Vereinigungsfreiheit. Unter Letztere fällt nicht nur das Recht, Vereinigungen wie eine Gewerkschaft zu gründen, sondern ebenfalls sich den Mitteln des Arbeitskampfs wie dem Streik zu bedienen. ${ }^{8}$ Der EGMR hat in jüngster Zeit mehrfach entschieden, dass nicht nur das abstrakte Verbot des Streikrechts, sondern auch die daran verknüpfte Sanktionierung der Teilnahme an einem Streik im Einzelfall unter den Schutzbereich des Art. 11 EMRK fällt. ${ }^{9}$ Freilich gewährt Art. 11 EMRK kein absolutes Streikrecht. Die Ausübung dieser Rechte darf gemäß Art. 11 Abs. 2 S. 1 EMRK nur Einschränkungen unterworfen werden, die gesetzlich vorgesehen und in einer demokratischen Gesellschaft für die nationale oder öffentliche Sicherheit, zur Aufrechterhaltung der Ordnung oder zur Verhütung von Straftaten, zum Schutz der Gesundheit oder der Moral oder zum Schutz der Rechte und Freiheiten anderer notwendig sind. Dem stehen rechtmäßige Einschränkungen der Ausübung dieser Rechte für Angehörige der Streitkräfte, der Polizei oder der Staatsverwaltung nicht entgegen. ${ }^{10}$

Nach der Rechtsprechung des EGMR verträgt sich ein generelles Verbot des Streiks für Beamte mit Art. 11 EMRK nicht. ${ }^{11}$ Es bedarf einer Differenzierung nach Beamtenkriterien bzw. konkreter Tätigkeit. Die Notwendigkeit des Streikverbots für Beamte müsse sich aus der konkreten Ausübung öffentlicher Aufgaben ergeben und diese Notwendigkeit lasse sich nicht allgemein für alle Beamten festlegen. ${ }^{12}$ Demnach ist es Auf-

7 EGMR, Urt. v. 21.4.2009 - 68959/01 = NZA 2010, 1423.

8 Meyer-Ladewig, in: Meyer-Ladewig, EMRK- Kommentar, 3. Auflage (2011), Art. 11, Rdnr. 13.

9 EGMR, Urt. vom 27.3.2007-Nr. 6615/03; Urt. v. 15.9.2009-Nr. 30946/04; Urt. v. 13.7.2010 - Nr. 33322/07.

10 Vgl. Art. 11 Abs. 2 S. 2 EMRK.

11 Zur Änderung früherer Rechtsprechung vgl. Niedobitek, ZBR 2010, 361 (367).

$12 E G M R$, Urt. v. 21.4.2009 = NZA 2010, 1423. 
gabe des Gesetzgebers, Kriterien für ein differenziertes Streikverbot festzulegen, die die Notwendigkeit eines Verbots rechtfertigen.

\section{Rechtsnatur der EMRK}

Anders als das Unionsrecht ist die EMRK ein klassischer völkerrechtlicher Vertrag zwischen den Konventionsstaaten über die Achtung der in der Konvention garantierten Menschenrechte. Die EMRK ist durch das Zustimmungsgesetz ins deutsche Recht transformiert worden. ${ }^{13}$ Die Gesetzesbindung nach Art. 20 Abs. 3 GG fordert die Berücksichtigung der Menschenrechte der Konvention im Rahmen methodisch vertretbarer Gesetzesauslegung. ${ }^{14}$ Zwar hat sie den Rang eines einfachen Gesetzes, ${ }^{15}$ dennoch ist auch das Grundgesetz im Lichte der EMRK auszulegen, denn das Grundgesetz hat eine generell völkerrechtsfreundliche Tendenz, die der Präambel, Art. 25, 24 Abs. 3 und Art. 9 Abs. 2 GG zu entnehmen ist. ${ }^{16}$ Daher ist die EMRK bei der Auslegung der Grundrechte des Grundgesetzes von deutschen Gerichten heranzuziehen. ${ }^{17}$

\section{Die Bedeutung der EGMR-Rechtsprechung}

Nun fragt sich, welche Auswirkung dann die Rechtsprechung des EGMR hat. Die Urteile des EGMR gelten zwar gemäß Art. 46 Abs. 1 EMRK inter partes, dennoch legen sie die Reichweite und Bedeutung der betreffenden Menschenrechte fest und geben die Auslegung der Rechte der Konvention durch den hierzu ermächtigten EGMR wieder, die auch in einem nicht am Verfahren beteiligten Staat von Bedeutung sein können. ${ }^{18}$ Eine Grundlage für die Beachtung der Entscheidungen des EGMR bildet Art. 1 EMRK, der die Konventionsstaaten verpflichtet, die Konventionsrechte zu gewährleisten. Sie sind zu gewährleisten in der Gestalt, die sich in der Rechtsprechung des EGMR entwickelt hat. ${ }^{19}$ Die Rechtsprechung des EGMR dient wie der Konventionstext als Auslegungshilfe für die Bestimmung von Inhalt und Reichweite von Grundrechten und rechtstaatlichen Garantien des GG, sofern diese nicht zur Einschränkung oder Minderung des Grundrechtsschutzes nach dem GG führt. ${ }^{20}$ Das BVerfG zieht es nicht in Zweifel, dass die Entscheidungen des EGMR den aktuellen Entwicklungsstand der Konvention widerspiegeln und daher eine besondere Bedeutung für das Konventionsrecht haben. ${ }^{21}$ Auch das BVerwG sieht in der ständigen Rechtssprechung des EGMR eine normative Leitfunktion, an der sich die Vertragsstaaten orientieren müssen. ${ }^{22}$ Damit

13 Die EMRK v. 4. 11. 1950, BGB1. 1952 II S. 685; Neubekanntmachung in der ab 01. 6. 2010 geltenden Fassung, BGB1. II S. 1198.

14 BVerfGE 111, 307 (317) = NJW 2004, 3407.

15 Degenhart, Staatsrecht I, 27. Auflage (2011), Rdnr. 253.

$16 \quad B V \operatorname{erfGE} 75,1$ (17) = NJW 1981, 2155; 111, 307 (317) = NJW 2004, 3407.

17 BVerfGE 74, 358 (370) = NJW 1987, 2427; 82, 106 (120) = NJW 1990, 2741.

18 Meyer-Ladewig, in EMRK- Kommentar (s. Fußn. 8), Art. 46 EMRK, Rdnr. 14.

19 Meyer-Ladewig, in EMRK- Kommentar (s. Fußn. 8), Art. 46 EMRK, Rdnr. 15.

20 Vgl. BverfGE 83, 119(128) = NJW 1991, 1043.

21 BVerfGE 111, $307=$ NJW 2004, 3407 (3409).

22 BVerwG, NVwZ 2002, 87. 
hat die Rechtsprechung des EGMR den gleichen Rang wie die EMRK selbst und für sie gilt das oben Gesagte gleichfalls.

\section{Verwaltungsgerichtsentscheidungen}

Im Folgenden werden die Entscheidungen des Verwaltungsgerichts Osnabrück und Verwaltungsgerichts Düsseldorf in den Blick genommen und untersucht.

\section{Sachverhalt ${ }^{23}$}

Die Klägerin steht als Lehrerin im Schuldienst des beklagten Bundeslandes. Sie ist seit September 2002 auf Lebenszeit verbeamtet und bisher strafrechtlich oder disziplinar nicht aufgefallen. Mit der Verfügung von 10.8.2009 wurde gegen die Klägerin anlässlich ihrer Teilnahme an einem Warnstreik am 28.9.2009 ein Disziplinarverfahren eingeleitet. Daraufhin wurde ordnungsgemäß eine Disziplinarverfügung, die der Klägerin eine Geldbuße in Höhe von $1.500 €$ auferlegte, erlassen. Dagegen hat sie vor dem zuständigen Gericht Klage erhoben.

\section{Entscheidung des VG Düsseldorf}

Das VG Düsseldorf hält an dem geltenden Streikverbot fest: Zwar stehe den Beamten nach dem nationalen Recht kein Streikrecht zu, ${ }^{24}$ ein solches Recht könne sich aus Art. 11 EMRK ergeben. Gemessen an der Anforderung des EGMR sei das Verbot des Beamtenstreiks mit der EMRK nicht vereinbar, denn es schließe Streik von Beamten generell aus, ohne eine Unterscheidung nach bestimmten Kategorien von Beamten vorzunehmen. ${ }^{25}$ Dies ändere aber nichts daran, dass das deutsche Beamtenrecht weiterhin gültig sei. ${ }^{26}$ Es lasse sich nicht im Wege völkerrechtsfreundlicher Auslegung beamtenrechtlicher Vorschriften an die EMRK anpassen, denn ihr stehen die hergebrachten Grundsätze des Berufsbeamtentums entgegen, damit würden die Grenzen der Auslegung überschritten. Es sei Aufgabe des verfassungsändernden Gesetzgebers, einen mit Art. 11 EMRK vereinbaren Rechtszustand im deutschen Beamtenrecht herbeizuführen. ${ }^{27}$

Das Gericht sieht zwar ein Dienstvergehen im Sinne des $\S 47$ Abs. 1 BeamtStG als gegeben an. Dies begründet es damit, dass die Klägerin ,während der Dienstzeit vorsätzlich an Warnstreiks teilgenommen und damit den Dienst versäumt hat. " Damit habe

23 VG Düsseldorf, Urt. v. 15.12.2010 - 31 K 3904/10.0; VG Osnabrück, Urt. v. 19.8.2011 - 9 A $1 / 11$ und 9 A 2/11.

24 VG Düsseldorf, Urt. v. 15.12.2010 - 31 K 3904/10.0, juris, Rdnr.13; Vgl. BVerfGE 8, 1(17) $=$ NJW 1958, 1228.

25 VG Düsseldorf, Urt. v. 15.12.2010 - 31 K 3904/10.0, juris, Rdnr.18.

26 VG Düsseldorf, Urt. v. 15.12.2010 - 31 K 3904/10.0, juris, Rdnr.19.

27 VG Düsseldorf, Urt. v. 15.12.2010 - 31 K 3904/10.0, juris, Rdnr.20; Ebenso Seifert, KritV 2009, S. 357 (375). 
sie schuldhaft die ihr obliegenden Pflichten verletzt. ${ }^{28}$ Der Beklagte dürfte allerdings auf das Dienstvergehen der Klägerin nicht mit dem Erlass einer Disziplinarverfügung reagieren, denn für die Sanktionierung der Teilnahme an einem Streik fehle es an einer Rechtfertigung im Sinne des Art. 11 Abs. 2 EMRK, da sie in einer demokratischen Gesellschaft nicht notwendig gewesen sei. ${ }^{29} \mathrm{Da}$ die Lehrer nicht zu den in Art. 11 Abs. 2 EMRK genannten Gruppen von Staatsbediensteten (Streitkräfte, Polizei, Staatsverwaltung) gehörten, ${ }^{30}$ käme eine Einschränkung des Streikrechts auch nicht allein wegen der Zugehörigkeit zu diesen Gruppen in Betracht. ${ }^{31}$ Eine Disziplinarmaßnahme sei gemäß $\S 33$ Abs. 1 Nr.4 LDG NRW aus, ,sonstigen Gründen, nämlich wegen des Verstoßes gegen die EMRK unzulässig gewesen, denn es hätte nicht die Gefahr der Funktionsunfähigkeit des öffentlichen Dienstes aufgrund von Streikmaßnahmen in erheblichem Umfang bestanden und damit sei die Disziplinarverfügung nicht notwendig gewesen." ${ }^{32}$ Mithin hält das VG Düsseldorf, das einen differenzierenden Ansatz zugrunde legt, die Disziplinarverfügung mit Art. 11 EMRK für unvereinbar.

\section{Entscheidung des VG Osnabrück}

Das VG Osnabrück hat sich in einem ähnlich gelagerten Fall anders entschieden und sich der Entscheidung des VG Düsseldorf nicht angeschlossen. Demzufolge ist die Disziplinarverfügung mit der Auferlegung der Geldbuße rechtmäßig ergangen. Dies wird damit begründet, dass den Beamten aufgrund geltender hergebrachter Grundsätze des Berufsbeamtentums schon kraft der Verfassung kein Streikrecht zusteht. Etwas anderes ergebe sich auch nicht aus Art. 11 EMRK und der Rechtsprechung des EGMR. Das VG Osnabrück setzt sich mit dem Urteil des EGMR auseinander. Zum einen führt es aus, dass der Begriff des „functionnaire“ nicht gleichzusetzen sei mit dem Begriff des Beamten und könne unter anderen Angehörige des öffentlichen Dienstes umfassen, ${ }^{33}$ zum anderen weist es auf die Entscheidungen des BVerfG in der Folgezeit hin, das an seiner bestehenden Rechtsprechung festhält. ${ }^{34}$ Das VG Osnabrück räumt zwar ein, es spreche vieles dafür, dass ein absolutes Streikverbot für Beamte dem Art. 11 EMRK widerspreche, dennoch hält es daran fest.

Eine solche funktionsbezogene Differenzierung, wie dies der EGMR verlange, sei mit Art. 33 Abs. 5 GG nicht vereinbar. Zu einer solchen Veränderung der Reichweite des Art. 33 Abs. 5 GG sei nur das BVerfG bzw. der verfassungsändernde Gesetzgeber befugt. ${ }^{35}$ Das Gericht sieht sich als staatlicher Funktionsträger gemäß Art. 31 Abs. 1 GG an die Entscheidungen des BVerfG gebunden.

28 VG Düsseldorf, Urt. v. 15.12.2010 - 31 K 3904/10.0, juris, Rdnr. 11.

29 VG Düsseldorf, Urt. v. 15.12.2010 - 31 K 3904/10.0, juris, Rdnr. $23 \mathrm{f}$.

30 EGMR, Urt. v. 8.12.1999 - Nr. 28541/95 = NVwZ 2000, 661 (663).

31 VG Düsseldorf, Urt. v. 15.12.2010 - 31 K 3904/10.0, juris, Rdnr. 26.

32 VG Düsseldorf, Urt. v. 15.12.2010 - 31 K 3904/10.0, juris, Rdnr. 35.

33 VG Osnabrück, Urt. v. 19.8.2011- 9 A 1/11, juris, Rdnr. 38.

34 VG Osnabrück, Urt. v. 19.8.2011- 9 A 1/11 juris, Rdnr. 30, 32.

35 VG Osnabrück, Urt. v. 19.8.2011-9 A 1/11 juris, Rdnr. 38. 


\section{Analyse der Entscheidungen}

Gemessen an den Anforderungen des EMGR bedarf es eines nach Funktionen differenzierenden Ansatzes. Selbst wenn man davon ausgeht, dass das deutsche Rechtssystem eine solche Differenzierung kennt, ${ }^{36}$ ist die deutsche Differenzierung lediglich eine statusrechtliche Unterscheidung und keine funktionsbezogene. Interessant ist, wie die beiden Verwaltungsgerichte mit diesem konventionswidrigen Zustand umgehen.

Im Kern halten die beiden Entscheidungen an dem absoluten Streikverbot für Beamte fest, allerdings mit völlig unterschiedlichen Konsequenzen für die konkret zu entscheidenden Fälle.

Das VG Osnabrück sieht im Ergebnis die Disziplinarverfügung als rechtmäßig ergangen an. Es beruft sich unter anderem darauf, dass der EGMR in seinen Urteilen nicht den Beamten als solchen, sondern alle Angehörige des Öffentlichen Dienstes gemeint haben könnte. Dies ist aber sehr zweifelhaft. In den maßgeblichen Entscheidungen des EGMR wird durchgängig der Begriff ,functionnaire“ verwendet, der im Französischen nur dem Begriff des Beamten entspricht. ${ }^{37}$ Jeder Versuch, diese neue Rechtsprechung des EGMR durch eine weite Auslegung des Begriffs „functionnaire“38 in Schranken zu halten, ist zwar kreativ, aus folgenden Gründen ist ihr jedoch nicht zu folgen: Eine solche weite Auslegung ist erst durch die deutsche Übersetzung ermöglicht worden, ${ }^{39}$ die den „functionnaire“ mit „Angehörige des Öffentlichen Dienstes“ gleichsetzt. Für die Auslegung der EMRK ist aber nach der Schlussformel der EMRK nur die französische und englische Sprache maßgeblich. Da das Urteil des EGMR in französischer Sprache verfasst ist, ist für die Würdigung der Entscheidung des EGMR aufFranzösisch abzustellen. Im Französischen ist der „functionnaire“, wer unter Verleihung eines Dienstgrades auf Dauer im öffentlichen Dienst eingewiesen worden ist. ${ }^{40}$

Es kann auch nicht eingewandt werden, es handele sich nicht um einen Fall in Frankreich oder es gehe nicht um die Bedeutung des „functionnaire“ im französischen Recht, ${ }^{41}$ denn die für die Auslegung maßgebliche Sprache ist so zu verstehen, wie sie ist und kann nicht je nach verklagter Vertragspartei im Einklang mit dessen Rechtssystem umgedeutet werden. Das hätte nur Rechtsunsicherheit zur Folge. Zudem lässt sich der Entscheidung des EGMR selbst entnehmen, dass der EGMR mit „functionnaire“ den Beamten meint, denn wenn es um Angestellte geht, spricht er von ,travailleurs publics“42 bzw. ,employees“. ${ }^{43}$ Daraus kann man folgern, dass die Angestellten gerade nicht unter dem Begriff „functionnaire“ fallen.

36 So Lindner, DÖV 2011, 305 (308).

37 Vgl. die Übersetzung aus dem französischen Original: Buschmann/ Löscher, AuR 2009, 269 ff; Vgl. zum Beamtenbegriff im französischen Recht Müller, Verwaltungsgerichtlicher Rechtsschutz des Deutschen und Französischen Beamten - Eine Rechtsvergleichende Untersuchung, 1. Auflage (1975), S. 18 f; Von diesem Verständnis einhellig ausgehend: Niedobitek, ZBR 2010, 361; Löcher, AuR 2009, 229; Seifert, KritV 2009, 357.

38 So Lindner, DÖV 2011, 305.

39 Vgl. NZA 2010, 1423.

40 Art. 1 des ,Statut de la Fonction Publique“ vgl. Müller (o. Fußn. 37), S. 18 f.

41 Lindner, DÖV 2011, 305 (308 f).

$42 E G M R$, Urt. v. 21.4.2009 - Nr. 68959/01, Rdnr. $32=$ NZA 2010, 1423.

43 EGMR, Urt. v. 21.4.2009 - Nr. 68959/01, Rdnr. 32 = NZA 2010, 1423. 
Ganz anders hat das VG Düsseldorf im Ergebnis entschieden. Es beteuert zwar, dass sich ein Dienstvergehen aufgrund des sich aus dem GG ergebenden Streikverbots ergibt. Das möchte das VG Düsseldorf jedoch nicht mit einer disziplinarischen Maßnahme bestrafen, da hierfür im Hinblick auf die Entscheidung des EGMR und die EMRK keine Notwendigkeit bestünde. Durch den differenzierenden Ansatz trägt das VG Düsseldorf der Rechtsprechung des EGMR Rechnung. Dies scheint auf dem ersten Blick nicht konsequent oder wie das VG Osnabrück meint, widersprüchlich.

Das VG Osnabrück erblickt einen Widerspruch zwischen dem Grundgesetz und völkerrechtsfreundlicher Auslegung des Disziplinarrechts, wenn ein Dienstvergehen folgenlos bleibt. Dies ist aber eine vorschnelle Schlussfolgerung, die sich zugegebener Maßen leicht erschließt. Worin besteht aber tatsächlich die Inkonsequenz bzw. der Widerspruch?

Das Grundgesetz selbst sieht keine Ahndungs- bzw. Bestrafungsmechanismen vor, so dass ein Widerspruch zum Grundgesetz nicht besteht. Die disziplinarische Ahndung des Dienstvergehens ergibt sich aus einfachem Gesetz. Es scheint zunächst inkonsequent zu sein, ein Dienstvergehen einfachgesetzlich festzustellen, dies aber disziplinarrechtlich nicht zu verfolgen. Gerade dies wird vom Gesetz durch die Einstellungsverfügung ermöglicht. ${ }^{44}$ Das Gesetz sieht selber die Möglichkeit einer Einstellung vor, dies zeigt, dass nicht jedes Dienstvergehen disziplinarische Folgen haben muss. Ausnahmen sind durchaus erlaubt. Zwar ist die Einstellung eines Disziplinarverfahrens wegen anderweitiger Rechtsprechung des EGMR bisher nicht bekannt und mit bislang von $\S 33$ Abs. 1 Nr. 4 LDG NRW zu erfassenden Fällen nicht identisch. Die Verwendung unbestimmter Rechtsbegriffe durch den Gesetzgeber dient aber gerade dazu, nicht vorausgesehene vergleichbare Fälle zu erfassen. Unter Einstellung aus sonstigen Gründen fielen bisher auch allgemeine Rechtsgrundsätze wie Verwirkung und Vertrauensschutz. Für die EMRK als allgemeiner Rechtsgrundsatz des Völkerrechts darf nichts anderes gelten.

Diese Auffassung ist insofern vertretbar, als nach der Rechtsprechung des EGMR nicht nur das abstrakte Verbot des Streikrechts, sondern auch die daran geknüpfte Sanktionierung der Teilnahme an einem Streik im Einzelfall unter den Schutzbereich des Art. 11 EMRK fällt. ${ }^{45}$

Von dem verfassungsrechtlich gebotenen Streikverbot kann das Gericht nicht ohne eine Verfassungsänderung bzw. Änderung der Rechtsprechung des BVerfG ${ }^{46}$ abweichen. Das einfache Gesetz muss das Gericht, soweit die Verfassung nicht entgegensteht, im Lichte der Rechtsprechung des EGMR auslegen. Aus einem verfassungsrechtlichen Verbot ergibt sich nicht ohne weiteres eine verfassungsrechtliche disziplinarische Ahndung. Dies ist kein Widerspruch zum Grundgesetz, sondern Konsequenz der Beachtung der völkerrechtlichen Verpflichtungen.

$44 \S 33$ LDG NRW; §33 BDG.

45 EGMR, Urt. v. 27.3.2007-Nr. 6615/03; 15.9.2009-Nr. 30946/04; 13.7.2010-Nr. 33322/07.

46 Zu dieser Frage vgl. Seifert, KritV 2009, 357. 


\section{Schlussbemerkung}

Das VG Düsseldorf versuchte durch seine Entscheidung im Rahmen seiner Befugnisse die Vorgaben des EMRG konsequent umzusetzen. Das eigentliche Problem, nämlich die Divergenz zwischen der Rechtsprechung des EGMR und der des BVerfG bleibt aber bestehen. Es fragt sich, ob die Rechtsprechung des BVerfG angesichts der völkerrechtsfreundlichen Tendenz des Grundgesetzes verfassungsrechtlich haltbar ist. In diesem Sinne wäre eine Vorlage nach Art. 100 Abs. 1 GG denkbar gewesen.

Eins steht aber fest: Das Verbot des Beamtenstreiks ist mit Art. 11 EMRK nicht vereinbar. Die Bundesrepublik kann zwar zur Durchsetzung der Rechtsprechung des EGMR selbst im Falle eines Urteils gegen die Bundesrepublik nicht gezwungen werden, sie macht sich aber bei einer konventionswidriger Rechtspraxis gegenüber den Betroffenen schadenersatzpflichtig. Das begründet Verpflichtungen, die letztendlich von Steuerzahlern getilgt werden müssen. Ob der Gesetzgeber oder die höchste Rechtsprechung diesen konventionswidrigen Zustand zu beseitigen willens ist, bleibt abzuwarten. 